\title{
Minimization of dark counts in PureB SPADs for NUV/VUV/EUV light detection by employing a 2D TCAD- based simulation environment
}

Tihomir Knežević, Lis K. Nanver, Tomislav Suligoj

Tihomir Knežević, Lis K. Nanver, Tomislav Suligoj, "Minimization of dark counts in PureB SPADs for NUV/VUV/EUV light detection by employing a 2D TCAD-based simulation environment," Proc. SPIE 10912, Physics and Simulation of Optoelectronic Devices XXVII, 109120Y (26 February 2019); doi: $10.1117 / 12.2508829$ 


\title{
Minimization of dark counts in PureB SPADs for NUV/VUV/EUV light detection by employing a 2D TCAD-based simulation environment
}

\author{
Tihomir Knežević*a, b Lis K. Nanver ${ }^{\mathrm{b}}$, Tomislav Suligoj ${ }^{\mathrm{a}}$ \\ ${ }^{a}$ University of Zagreb, Faculty of Electrical Engineering and Computing, Micro and Nano \\ Electronics Laboratory, Croatia; ${ }^{b}$ University of Twente, Faculty of Electrical Engineering \\ Mathematics \& Computer Science, Enschede, The Netherlands
}

\begin{abstract}
PureB single-photon avalanche diodes (SPADs) were investigated with the aid of a newly developed TCAD-based numerical modeling method with which characteristics related to the avalanching behavior can be simulated. The $\mathrm{p}^{+}$region forming the anode of the PureB $\mathrm{p}^{+} \mathrm{n}$ photodiode is extremely shallow, only a few nanometer deep, which is essential for obtaining a high photon detection efficiency (PDE) for near-, vacuum- and extreme-ultraviolet (NUV/VUV/EUV) light detection but when an implicit guard ring (GR) is implemented, the dark count rate (DCR) can, despite the GR, be deteriorated at the very sharp corners of the $\mathrm{p}^{+}$-region where there is a high concentration of the electric-field. By comparing measurements to simulations, the main mechanism dominating the DCR in the PureB SPADs was identified as band-toband tunneling (BTBT) while trap-assisted-tunneling also plays a role when the perimeter breakdown is low. Increasing the dose of carriers in the enhancement region negatively impacts the total DCR of the device, but also shifts the origin of the dominant DCR contribution from perimeter to the active region. The simulations for optimization of the SPAD geometry predict that a modification of the n-doped epitaxial region of the PureB SPADs could decrease the DCR by almost two orders of magnitude. This is achieved by increasing the n-epi-layer thickness from $1 \mu \mathrm{m}$ to $3 \mu \mathrm{m}$ and lowering the doping from $10^{15} \mathrm{~cm}^{-3}$ to $10^{14} \mathrm{~cm}^{-3}$. A high electric field at the vertical pn junction in the active region can also be minimized by modifying the implantation parameters of the n-enhancement region thus keeping the BTBT contribution to the DCR sufficiently low.
\end{abstract}

Keywords: photodiode, single-photon avalanche diodes (SPADs), detectors, silicon, pure boron, guard rings, avalanche breakdown, band-to-band tunneling, trap-assisted tunneling

\section{INTRODUCTION}

Detection of ultraviolet (UV) light with Si photodiodes in the near-, vacuum- and extreme-ultraviolet (NUV/VUV/EUV) wavelength ranges requires the photosensitive region to be located very close to the Si surface since the light absorption length is low, even as low as $5 \mathrm{~nm}$ in the $200 \mathrm{~nm}$ to $300 \mathrm{~nm}$ wavelength range. Likewise, low-energy electrons with energies below $1 \mathrm{keV}$ penetrate the silicon surface by only a few nanometer. Pure amorphous boron (PureB) technology breaks the trade-off between the depth of the photosensitive region, hence the sensitivity, and low dark current in Si photodiodes. At the interface between Si and a deposited B-layer, a charged layer is formed that in itself gives a lowsaturation-current $\mathrm{p}^{+} \mathrm{n}$-like junction that can be less than $10 \mathrm{~nm}$ deep when the deposition is performed at a temperature of $400{ }^{\circ} \mathrm{C}$ up to $700^{\circ} \mathrm{C}^{1}$. When applied as the anode of Si photodiodes these PureB diodes offer low dark currents comparable to deeply diffused pn junction devices ${ }^{2}$ as well as high stability and robustness during high-dose and high-energy radiation/particle exposure ${ }^{2,3}$. The almost ideal and reliable responsivity has been pivotal for the use of PureB layers in semiconductor detector devices for both NUV/VUV/EUV light ${ }^{2}$ and low-energy electrons ${ }^{4}$ detection. Research is also directed towards fabrication of PureB detectors for high-sensitivity in UV photon starved environments ${ }^{5}$ or at low-radiation levels of electrons ${ }^{6}$.

Single photon resolution in a light-deprived environment can be achieved by using avalanche photodiodes that can be reversely biased with a voltage $V_{\mathrm{D}}$ above the breakdown voltage ( $\left.V_{\mathrm{BR}}\right)$ to an excess bias of $V_{\mathrm{EX}}=V_{\mathrm{D}}-V_{\mathrm{BR}}$, without directly triggering avalanche breakdown. In this regime, even a single photon can trigger a self-sustaining impact ionization process that increases the current of the device until the applied voltage is reduced to below $V_{\mathrm{BR}}$ by external circuitry designed to quench the avalanche ${ }^{7}$. The output of these single-photon avalanche diodes (SPADs) are pulses or counts which are *tihomir.knezevic@ fer.hr; phone +385 1 6129-564; minel.fer.hr 
correlated to the number of incoming photons. The photon detection efficiency (PDE) is a measure for the quality of the SPAD and is defined as the ratio of the number of detected photons to incoming photons. The SPAD can also be triggered by unwanted processes such as thermally generated carriers, tunneling currents and background photons, causing a socalled dark count rate (DCR) which adds to the noise of the device. Shockley-Read-Hall (SRH) processes dominate the thermal carrier generation while common tunneling processes include band-to-band tunneling (BTBT) and/or trap-assisted tunneling (TAT). Single-photon detection is exploited in various applications and at different wavelengths such as: medical diagnostics using time-of-flight (TOF) positron emission tomography (PET) where $511 \mathrm{keV}$ gamma rays are detected ${ }^{8}$, light detection and ranging (LiDAR) using the $905 \mathrm{~nm}^{\text {light }}{ }^{9}$, and deep-space laser communication operated at $1.064 \mu \mathrm{m}^{10}$. SPADs for UV radiation detection are utilized in, for example, non-line-of-sight ultraviolet communication systems ${ }^{11,12}$.

In all applications, it is of paramount importance to have a high PDE at the wavelengths of interest while keeping the DCR sufficiently low. Only recently did modeling of avalanche breakdown probability according to the model of McIntyre ${ }^{13}$ become available in Technology Computer-Aided Design (TCAD) software such as Sentaurus Device ${ }^{14}$. Information on the electron and hole avalanche breakdown probability is useful in simulation of SPADs, but a direct calculation of the discrete properties such as DCR or PDE is still not available using Sentaurus Device. Therefore, we developed a TCADbased simulation environment capable of simulating 2D DCR characteristics of Si SPADs ${ }^{15}$ based on avalanche probability calculations in $1 \mathrm{D}^{16}$ following the methods of Oldham et al. ${ }^{17}$. In this paper, the developed extension is used to study and analyze DCR contributions in experimentally fabricated PureB SPADs with implicit GRs. In general, GRs ensure that the peripheral breakdown, $V_{\mathrm{BR} \text {,per, }}$, is higher than $V_{\mathrm{BR}}$. An implicit GR is commonly chosen to achieve a compact device and high fill factor in arrays. Moving the breakdown away from the perimeter also has the advantage of avoiding a high field at the $\mathrm{SiO}_{2}$ interface that otherwise is a source of defect-induced carrier generation. By fitting to measurement results, the sources of DCR in PureB SPADs were identified and the critical regions in the device structures were located. With this knowledge, the fabrication parameters were numerically optimized showing that the DCR would be reduced by small structural adjustments.

\section{SOURCES OF DCR IN PUREB SPADS}

\subsection{PureB SPAD process-simulations}

Simulations of the PureB SPAD fabrication steps ${ }^{18}$ are performed using Sentaurus Process ${ }^{19}$. The structure consists of an $\mathrm{n}^{+}$buried layer simulated with a constant phosphorus doping concentration of $10^{18} \mathrm{~cm}^{-3}$ on top of which a $1-\mu \mathrm{m}$-thick epitaxial region $\left(t_{\mathrm{epi}}\right)$ is deposited with a phosphorus doping concentration of $N_{\mathrm{epi}}=10^{15} \mathrm{~cm}^{-3}$. The active part of the device and the implicit GR configuration is defined by an n-enhancement region. A two-step implantation process is performed through a 30-nm-thick thermal oxide layer. The first implantation is done at an energy of $40 \mathrm{keV}\left(E_{\mathrm{e} 1}\right)$ to a varied dose of $1 \times 10^{12} \mathrm{~cm}^{-2}$ to $8.5 \times 10^{12} \mathrm{~cm}^{-2}\left(Q_{\mathrm{e} 1}\right)$, while the second implantation step was done at $300 \mathrm{keV}\left(E_{\mathrm{e} 2}\right)$ to a dose of $5 \times 10^{12} \mathrm{~cm}^{-2}$ $\left(Q_{\mathrm{e} 2}\right)$. Annealing of the implantation was performed at $950{ }^{\circ} \mathrm{C}$ for $20 \mathrm{~min}$. The anode region is formed by a PureB deposition at $700{ }^{\circ} \mathrm{C}$ for 6 min which resulted in a 2.5 -nm-thick layer that was annealed at $850{ }^{\circ} \mathrm{C}$ for 1 min. A schematic cross section of the PureB SPAD is given in Figure 1 (a) while the simulated doping profile in the active region is shown in Figure 1 (b).

(a)

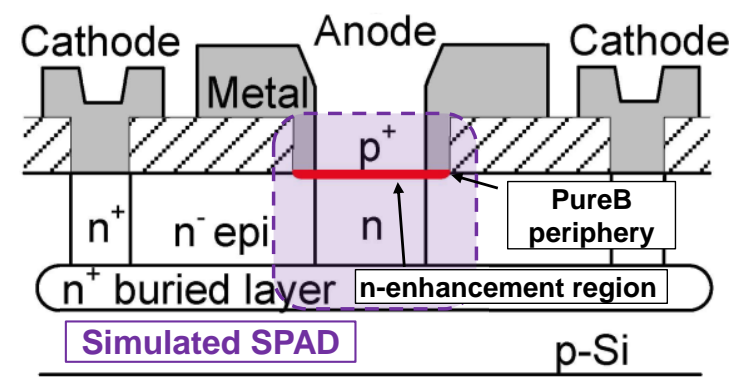

(b)

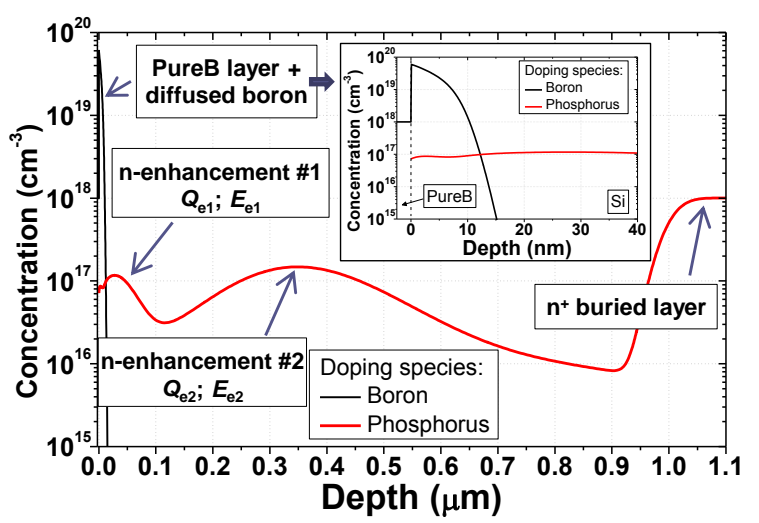

Figure 1. (a) Cross section of a PureB SPAD indicating the simulated region. (b) Doping concentration profile after all the simulated processing steps. Inset: Close-up of the doping concentration profile near the interface indicating the pn-junction depth. 


\subsection{Calculation of dark count rate}

In this work, the simulations of DCR and PDE are made possible in Sentaurus Device by following the methods of Oldham et al. ${ }^{17}$ to develop a TCAD-based simulation environment where probabilities are calculated for the event that an electron $\left(P_{\mathrm{e}}\right)$ or hole $\left(P_{\mathrm{h}}\right)$ will cause an avalanche when injected into the high-field region from any position, $x$, in the structure. The procedure requires extraction of the ionization coefficients at each given $V_{\mathrm{Ex}}$. These are then used as an input parameter for solving a set of differential equations derived in ${ }^{17}$. This yields $P_{\mathrm{e}}$ and $P_{\mathrm{h}}$ of the device in the 1D case. In addition, we have expanded the simulation procedure to calculate the DCR of both $2 \mathrm{D}$ and $3 \mathrm{D}$ devices ${ }^{15}$ by positioning the $1 \mathrm{D}$ crosssection throughout the $2 \mathrm{D}$ or $3 \mathrm{D}$ structure in a manner that correctly represents the potential gradients of importance for the carrier transport. Using a 2D DCR calculation method and simulations performed in a cylindrical coordinate system it is possible to calculate the total DCR of devices with a circular anode without the need for tedious 3D simulations. Carrier generation rates obtained from TCAD simulations are combined with calculated avalanche probabilities at various cross sections of the device to yield a DCR contribution at a certain position in the structure ${ }^{16}$. This procedure was used to simulate the total DCR of the experimental PureB SPADs. In PureB SPADs, the dominant dark carrier generation mechanisms were identified as SRH, BTBT and $\mathrm{TAT}^{15}$.

\subsection{Analysis of DCR in PureB SPADs}

The structure obtained from the process simulations is fed into Sentaurus Device to perform 1D and 2D device simulations. Impact ionization coefficients from the University of Bologna impact ionization model ${ }^{20}$ are used for simulating avalanche generation. Thermal generation is modeled by SRH with parameters of the electron and hole lifetime set to $2 \times 10^{-5} \mathrm{~s}$ while the position of the trap was $0.15 \mathrm{eV}$ from the middle of the bandgap towards the conduction band. These parameters yield the dark current values that were reported previously for PureB photodiodes ${ }^{2}$. The BTBT and TAT, which dominate the dark current at high electrical fields, are modeled using the non-local tunneling models available in Sentaurus Device ${ }^{14}$. The parameter $B$ of the BTBT model from Sentaurus Device was varied within $10 \%$ of the default value to account for variations in the processing conditions that may influence the BTBT. The traps at the anode periphery of the implicit GR are modeled with a concentration of $10^{11} \mathrm{~cm}^{-3}$, cross-section of $10^{-15} \mathrm{~cm}^{2}$, and the position of the trap at $0.3 \mathrm{eV}$ from the middle of the bandgap towards the conduction band. The breakdown voltage of the active region $\left(V_{\mathrm{BR} \text {,act }}\right)$ is simulated in 1D for PureB SPADs with $Q_{\mathrm{e} 1}$ equal to $1 \times 10^{12} \mathrm{~cm}^{-2}, 3.5 \times 10^{12} \mathrm{~cm}^{-2}, 6 \times 10^{12} \mathrm{~cm}^{-2}$ and $8.5 \times 10^{12} \mathrm{~cm}^{-2}$. Measured ${ }^{18}$ and simulated breakdown voltages are compared in Figure 2 for different $Q_{\mathrm{e} 1}$ and the results show excellent agreement confirming that the impact ionization starts in the active region.

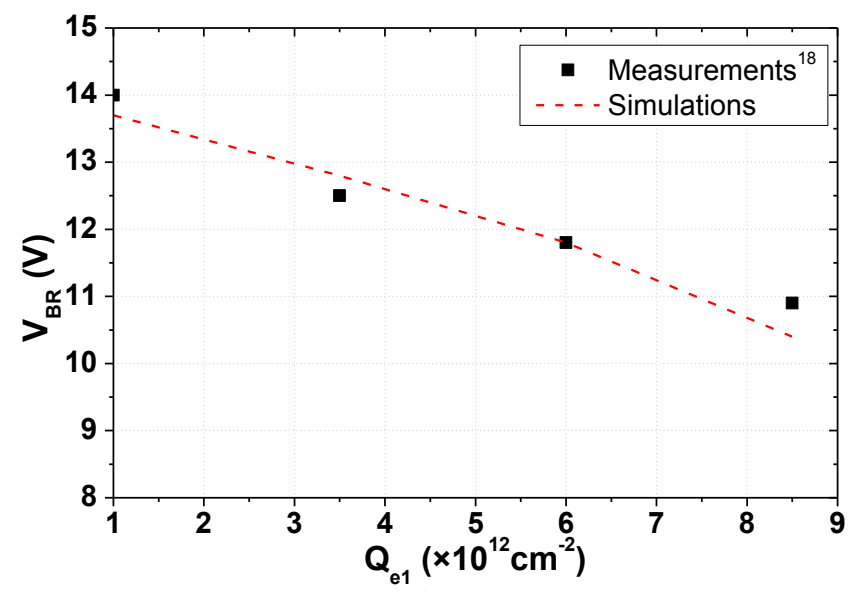

Figure 2. Comparison of the measured $V_{\mathrm{BR}}$ of PureB SPADs ${ }^{18}$ and simulated $V_{\mathrm{BR}}$ of the $1 \mathrm{D}$ active region obtained from process simulations with $Q_{\mathrm{e} 1}=1 \times 10^{12} \mathrm{~cm}^{-2}, 3.5 \times 10^{12} \mathrm{~cm}^{-2}, 6 \times 10^{12} \mathrm{~cm}^{-2}$ and $8.5 \times 10^{12} \mathrm{~cm}^{-2}$.

Perimeter breakdown $\left(V_{\mathrm{BR}, \mathrm{per}}\right)$ at the PureB periphery is simulated by omitting the enhancement region implantation. It is found that $V_{\mathrm{BR}, \mathrm{per}}$ can be as low as $14 \mathrm{~V}$ since it depends on the structural and geometrical parameters such as oxide thickness, electrode overlap, lateral boron diffusion, and oxide interface charge concentration. A positive oxide interface charge is set to $4 \times 10^{11} \mathrm{~cm}^{-2}$ yielding $V_{\mathrm{BR} \text {,per }}$ of $16.1 \mathrm{~V}$. Such a low $V_{\mathrm{BR}, \text { per }}$ can then limit the performance of a PureB SPAD. When the SPAD is operated at $V_{\mathrm{EX}}$ higher than $V_{\mathrm{BR} \text {,per }}-V_{\mathrm{BR} \text {,act }}$ an additional DCR source at the periphery starts to dominate the total $\mathrm{DCR}^{15}$. BTBT was identified as the main mechanism governing the DCR of the PureB SPAD with $Q_{\mathrm{e} 1}=$ $1 \times 10^{12} \mathrm{~cm}^{-2}$ at higher $V_{\mathrm{EX}}$, while at lower voltages, contribution from TAT can also be important. At $V_{\mathrm{EX}}$ below $2 \mathrm{~V}, \mathrm{DCR}$ 
is dominated by SRH ${ }^{15}$ events. Contributions to the DCR from BTBT at certain positions in the detector were identified and the results for $Q_{\mathrm{e} 1}=1 \times 10^{12} \mathrm{~cm}^{-2}$ and $8.5 \times 10^{12} \mathrm{~cm}^{-2}$ at $V_{\mathrm{EX}}=5 \mathrm{~V}$ are given in Figure 3. The parameter $B$ was set to $2.7 \times 10^{7} \mathrm{~V} / \mathrm{cm}$. For $Q_{\mathrm{el}}=1 \times 10^{12} \mathrm{~cm}^{-2}$ the level of BTBT is 5 orders of magnitude higher at the periphery than in the central n-enhancement region. The breakdown at the PureB periphery introduces an additional DCR source which significantly increases the total DCR of the device: for $V_{\mathrm{EX}}=5 \mathrm{~V}$ the DCR from BTBT is $900 \mathrm{~Hz}$ while the n-enhancement region only delivers $3 \times 10^{-2} \mathrm{~Hz}$. Increasing the dose of the enhancement region decreases $V_{\mathrm{BR}}$ and negatively affects the DCR contribution from BTBT. With $Q_{\mathrm{e} 1}=8.5 \times 10^{12} \mathrm{~cm}^{-2}$ the $V_{\mathrm{BR}}$ is $10.6 \mathrm{~V}$ and the n-enhancement region becomes the dominant source of BTBT DCR.

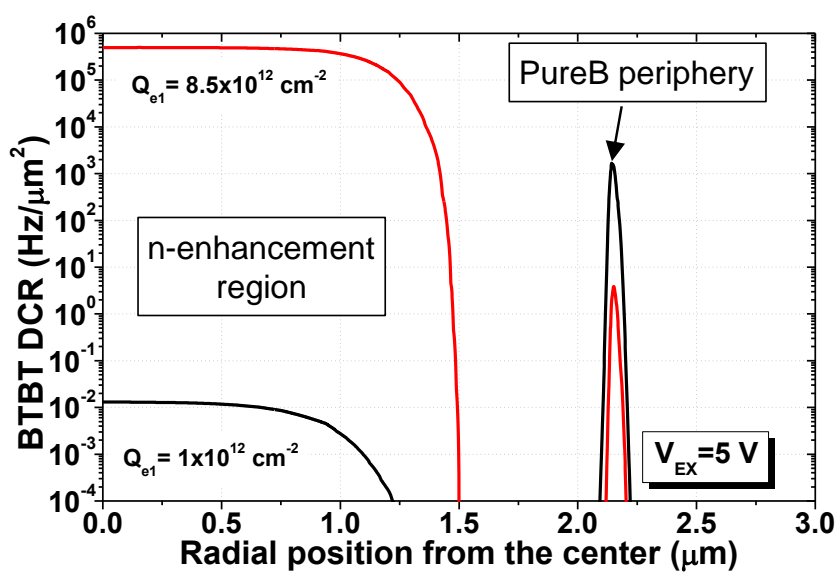

Figure 3. BTBT DCR extracted at radial positions from the center of the PureB SPAD anode at $V_{\mathrm{EX}}=5 \mathrm{~V}$. The radii of the anode and n-enhancement regions are $2 \mu \mathrm{m}$ and $1.5 \mu \mathrm{m}$, respectively.

The total DCRs as a function of $V_{\mathrm{EX}}$ are simulated for devices with $Q_{\mathrm{e} 1}$ between $1 \times 10^{12} \mathrm{~cm}^{-2}$ and $8.5 \times 10^{12} \mathrm{~cm}^{-2}$. The parameter $B$ was set to $2.7 \times 10^{7} \mathrm{~V} / \mathrm{cm}, 2.2 \times 10^{7} \mathrm{~V} / \mathrm{cm}, 2.4 \times 10^{7} \mathrm{~V} / \mathrm{cm}$ and $2.7 \times 10^{7} \mathrm{~V} / \mathrm{cm}$ for $Q_{\mathrm{e} 1}$ of $1 \times 10^{12} \mathrm{~cm}^{-2}, 3.5 \times 10^{12}$ $\mathrm{cm}^{-2}, 6 \times 10^{12} \mathrm{~cm}^{-2}$ and $8.5 \times 10^{12} \mathrm{~cm}^{-2}$, respectively. These values lie in the theoretical range between $1.9 \times 10^{7} \mathrm{~V} / \mathrm{cm}^{21}$ and $3.1 \times 10^{7} \mathrm{~V} / \mathrm{cm}^{22}$ and are used as a fitting parameters to account for possible process variations. The simulations are compared to the measurements ${ }^{18}$ as shown in Figure 4. There is a good agreement with the measurement although there is a difference in the slope at certain positions. There are several sources contributing the DCR and process non-uniformity can introduce additional sources which are not covered by simulations.

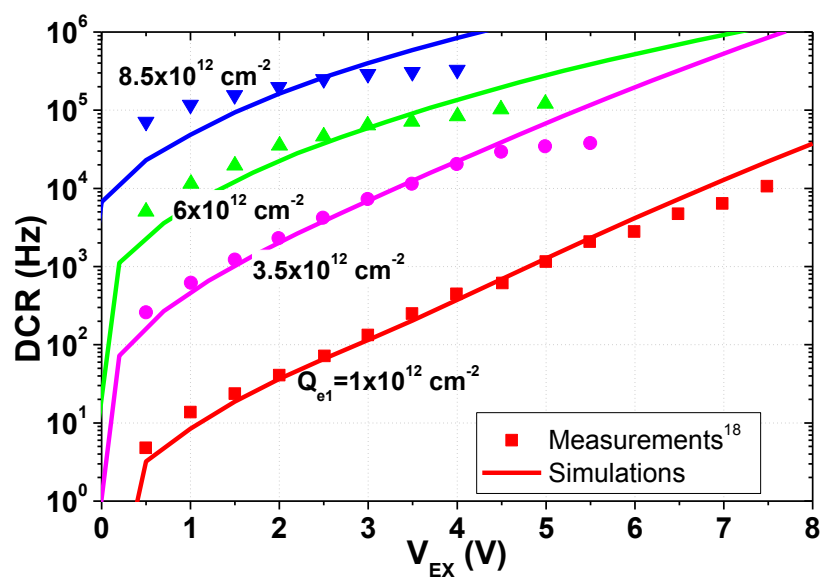

Figure 4. Comparison of the measured ${ }^{18}$ and simulated DCR as a function of $V_{\mathrm{EX}}$ for $Q_{\mathrm{e} 1}=1 \times 10^{12} \mathrm{~cm}^{-2}, 3.5 \times 10^{12} \mathrm{~cm}^{-2}$, $6 \times 10^{12} \mathrm{~cm}^{-2}$ and $8.5 \times 10^{12} \mathrm{~cm}^{-2}$.

The validity of the developed enhancement to the device simulator was further confirmed by calculating the SRH, BTBT and TAT contributions to the DCR in the temperature range from $220 \mathrm{~K}$ to $320 \mathrm{~K}$ for $V_{\mathrm{EX}}=6 \mathrm{~V}$. Temperature dependent models were used in the simulations. Comparison of the simulated total DCR to the measurements is given in Figure 5 and an excellent agreement is achieved. Components contributing to the DCR at different temperatures can be identified 
showing that BTBT dominates, while SRH is negligible at high $V_{\mathrm{EX}}$. SRH generation can easily be controlled by lowering the temperature since the DCR reduces from $400 \mathrm{~Hz}$ to only $10^{-3} \mathrm{~Hz}$ for a decrease in temperature from $320 \mathrm{~K}$ to $220 \mathrm{~K}$, while the TAT and BTBT have a much slower rate of reduction. For these, changing the device structure and processing parameters needs to be considered in order to decrease the DCR of the device.

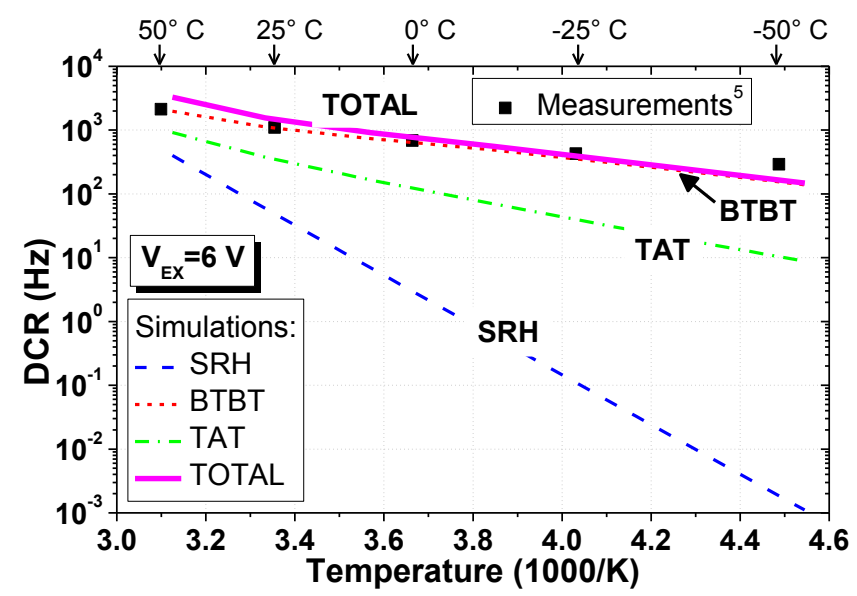

Figure 5. Comparison of the measured ${ }^{5}$ and simulated DCR as a function of temperature at $V_{\mathrm{EX}}=6 \mathrm{~V}$. Contributions of the BTBT, TAT and SRH DCR to the total DCR are indicated.

\section{DCR OPTIMIZATION}

\subsection{Minimization of tunneling induced DCR in PureB SPADs}

For PureB detectors to remain highly sensitive to light with wavelengths below $400 \mathrm{~nm}$, out-diffusion of the boron-doped $\mathrm{p}^{+}$anode region should be limited although this would help to reduce the electric field at the anode perimeter. Increasing the $V_{\mathrm{BR} \text {,per }}$ should therefore rather be achieved by modifying the $\mathrm{n}$-doped region. The options for doing this in the present device include increasing the distance to the buried $\mathrm{n}^{+}$layer, reducing the $\mathrm{n}$-epi doping, and changing the $\mathrm{n}$-enhancement implantation, all of which are examined by simulations. The simulated $V_{\mathrm{BR}, \text { per }}=16.1 \mathrm{~V}$ of the experimental device, was increased to $19.5 \mathrm{~V}$ and $18 \mathrm{~V}$ for $t_{\text {epi }}=3 \mu \mathrm{m}$ and $N_{\text {epi }}$ either equal to $10^{14} \mathrm{~cm}^{-3}$ or $10^{15} \mathrm{~cm}^{-3}$, respectively. For these two epidoping levels, the DCR contribution from SRH and BTBT at $V_{\mathrm{EX}}=5 \mathrm{~V}$ are shown in Figure 6 for increasing $t_{\text {epi }}$ values. The BTBT contribution can be reduced from $900 \mathrm{~Hz}$ to $20 \mathrm{~Hz}$ for $N_{\mathrm{epi}}=10^{14} \mathrm{~cm}^{-3}$ and $t_{\mathrm{epi}}=3 \mu \mathrm{m}$. With the proposed processing parameters, the DCR would be mainly determined by SRH that then limits the DCR to $\approx 50 \mathrm{~Hz}$. The SRH contribution has the advantage of being effectively lowered by improving the epitaxial layer quality or by reducing the operating temperature of the SPAD. At even lower $V_{\mathrm{EX}}$, the BTBT DCR from the perimeter will be significantly reduced and DCR will be generated only in the n-enhancement region of the SPAD.

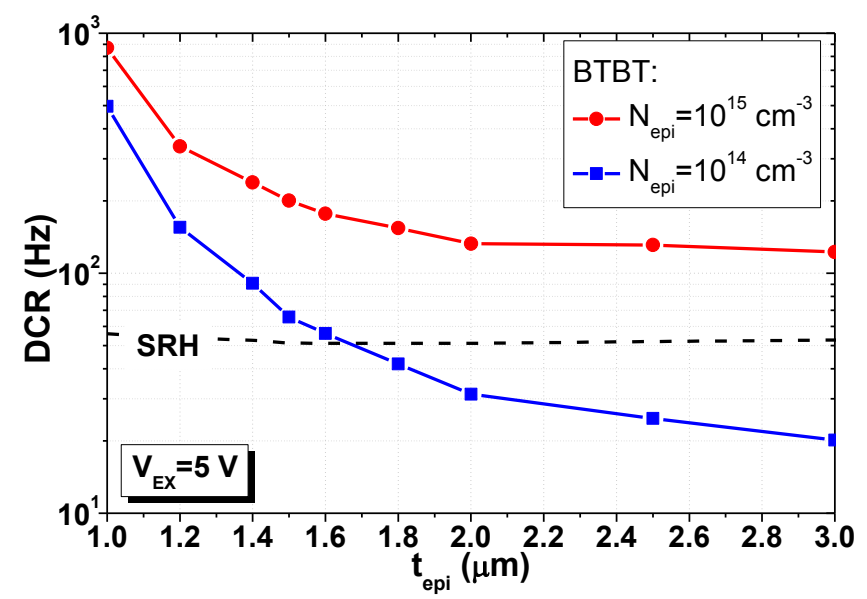

Figure 6. Reduction of DCR from BTBT as a function of epi-layer thickness for $N_{\mathrm{epi}}=10^{14} \mathrm{~cm}^{-3}$ and $10^{15} \mathrm{~cm}^{-3}$ at $V_{\mathrm{EX}}=5 \mathrm{~V}$. The DCR stemming from SRH events is shown for reference. 


\subsection{Optimization of DCR in the active region}

In cases where the $V_{\mathrm{BR}, \text { per }}$ is sufficiently higher than $V_{\mathrm{BR}}$, only the n-enhancement region determines the total DCR. 1D simulations of only this region were performed at $V_{\mathrm{EX}}=5 \mathrm{~V}$ for different temperatures to obtain the DCR per unit area from SRH or BTBT as shown in Figure 7. The SRH contribution dominates for temperatures down to $240 \mathrm{~K}$. In addition, in Figure 7 the $Q_{\mathrm{e} 2}$ is varied from $3 \times 10^{12} \mathrm{~cm}^{-2}$ to $5 \times 10^{12} \mathrm{~cm}^{-2}$. Decreasing the $Q_{\mathrm{e} 2}$ lowers the peak doping concentration of the n-enhancement implantation. The results show that the DCR from SRH does not change with $Q_{\mathrm{e} 2}$. In contrast, the BTBT DCR is significantly reduced by lowering the $Q_{\mathrm{e} 2}$. The steep $\mathrm{p}^{+} \mathrm{n}$ junction formed by the PureB deposition, introduces a large electric field at the pn-junction. This gives rise to high DCR values from BTBT which dominates especially at the lower temperatures. Nevertheless, the processing parameters which decrease the donor concentration near the pn-junction reduce the electric field and consequently also the DCR from BTBT.

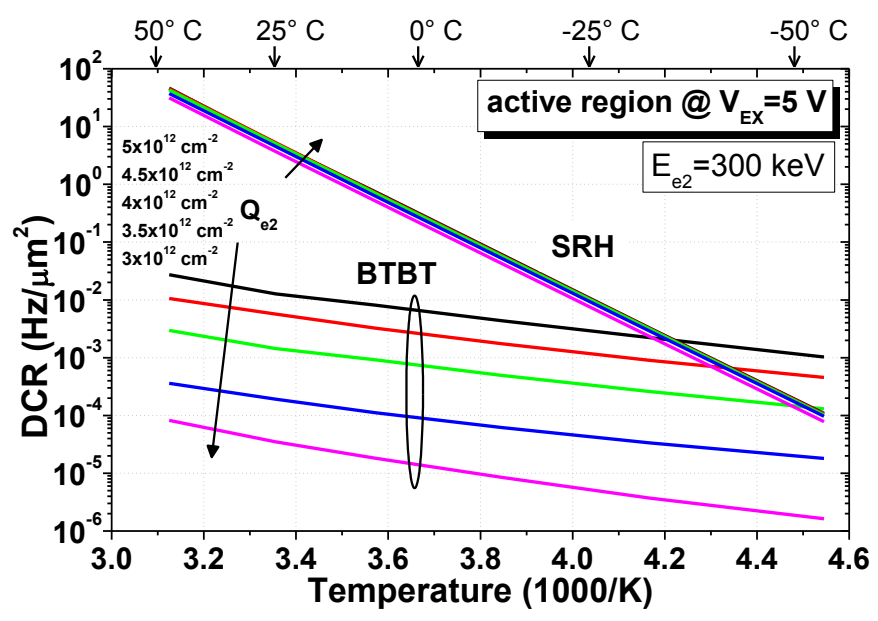

Figure 7. Impact of the $Q_{\mathrm{e} 2}$ on the DCR per unit area from SRH and BTBT in the active region of the PureB SPAD at $V_{\mathrm{EX}}=$ $5 \mathrm{~V}$ for temperatures between $220 \mathrm{~K}$ and $320 \mathrm{~K}$.

Special care should be applied during the critical fabrication steps such as epitaxial layer growth to eliminate inclusion of traps to the active region where high electric field is present due to the steep $\mathrm{p}^{+} \mathrm{n}$ junction. Both SRH and TAT depend on the number of traps introduced during the device processing and if these could be lowered, the BTBT could become the main source of DCR in the active region. In the PureB SPAD process, DCR BTBT can be lowered by optimization of the n-enhancement region by changing parameters of the first and the second implantation step. In Figure 8, DCR form BTBT in the active region at $V_{\mathrm{EX}}=5 \mathrm{~V}$ is plotted together with $V_{\mathrm{BR} \text {,act }}$ with respect to $E_{\mathrm{e} 1}$. Decreasing $E_{\mathrm{e} 1}$ from $40 \mathrm{keV}$ to $20 \mathrm{keV}$ can decrease the BTBT DCR in the active region by almost an order of magnitude while keeping the $V_{\mathrm{BR} \text {,act }}$ almost constant.

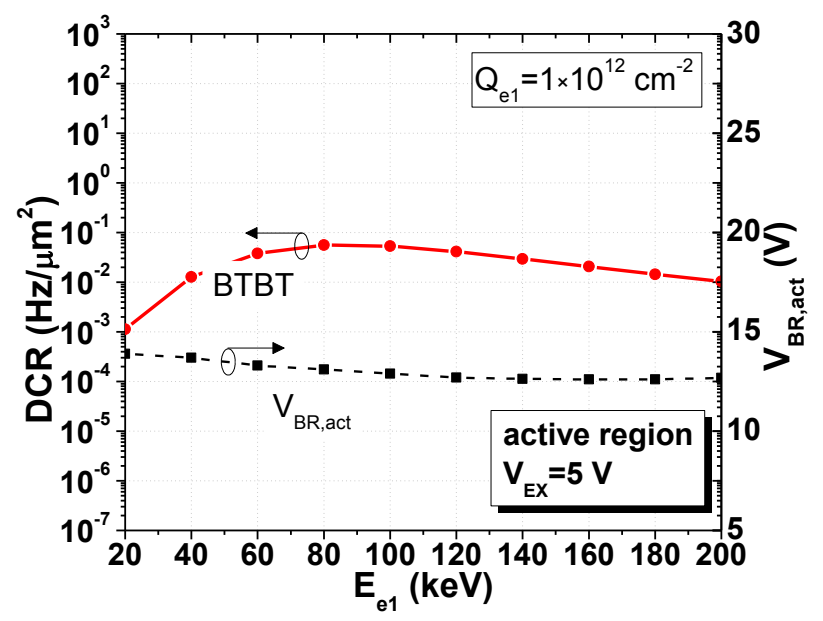

Figure 8. Impact of $E_{\mathrm{e} 1}$ on BTBT DCR in the active region of a PureB SPAD at $V_{\mathrm{EX}}=5 \mathrm{~V}$. The influence on $V_{\mathrm{BR} \text {,act }}$ is also plotted. 
Other than lowering $Q_{\mathrm{e} 1}$ or $E_{\mathrm{e} 1}$ which decreases the DCR, changing the processing parameters of the second implantation step such as $Q_{\mathrm{e} 2}$ and $E_{\mathrm{e} 2}$ impacts the BTBT DCR. Simulation results of the DCR from BTBT in the active region at $V_{\mathrm{EX}}=$ $5 \mathrm{~V}$ as a function of $Q_{\mathrm{e} 2}$ and $E_{\mathrm{e} 2}$ are shown in Figure 9 (a) and (b), respectively. Varying $Q_{\mathrm{e} 2}$ and $E_{\mathrm{e} 2}$ impacts $V_{\mathrm{BR}, \text { act }}$ which is also shown in the figures. Decreasing the dose in the n-enhancement region decreases the electric field at the pn-junction which decreases the BTBT DCR and increases the $V_{\mathrm{BR} \text {,act }}$. The implantation energy $E_{\mathrm{e} 2}$ can also be used to adjust the BTBT DCR since it shifts the position of the second peak thus influencing the electric field distribution. When $V_{\mathrm{BR} \text {,act }}$ is used as optimization constraint, simulations suggest that it is possible to adjust the implantation energy in order to have a lower BTBT DCR.

(a)

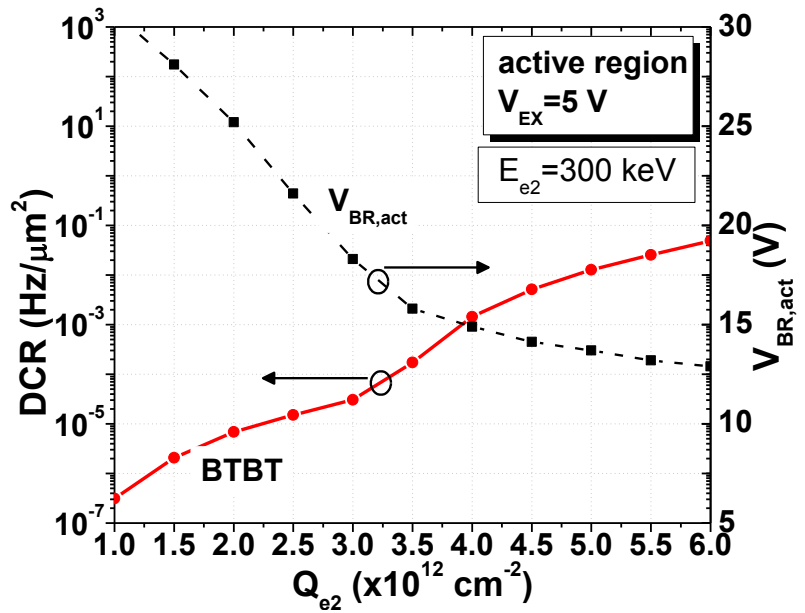

(b)

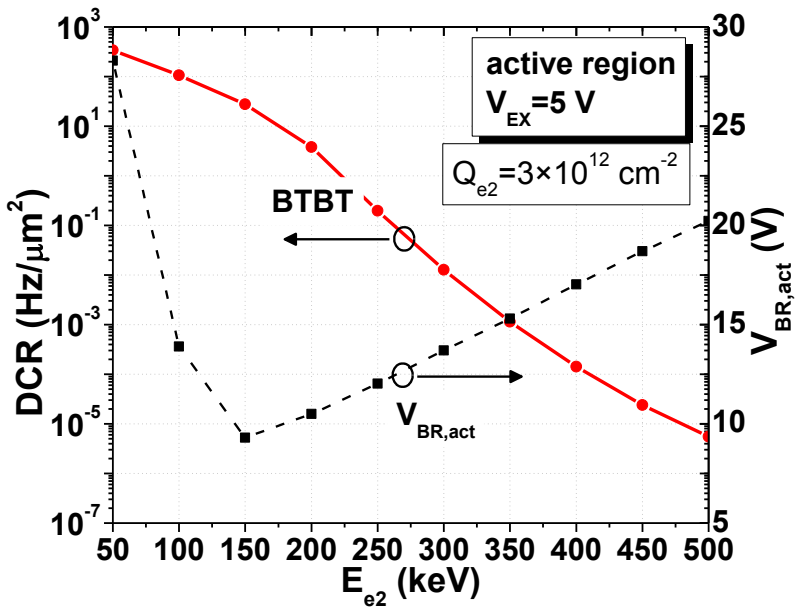

Figure 9. Impact of (a) $Q_{\mathrm{e} 2}$ and (b) $E_{\mathrm{e} 2}$ on BTBT DCR in the active region of a PureB SPAD at $V_{\mathrm{EX}}=5 \mathrm{~V}$. The influence on $V_{\mathrm{BR}, \text { act }}$ is also plotted.

\section{CONCLUSIONS}

The very steep PureB $\mathrm{p}^{+} \mathrm{n}$ junction, with sharp corners at the perimeter, results in high potential gradients that require careful design of the $\mathrm{n}$-doped region to prevent tunneling events from becoming a dominating source of dark counts. A newly developed simulation environment was used to analyze the behavior of experimentally fabricated PureB SPADs with an implicit guard ring created by a central n-enhancement region. The measured DCRs were successfully reproduced in the simulations. In these experimental devices the breakdown at the perimeter was shown to be so low that, at high $V_{\mathrm{EX}}$, avalanche events at the perimeter significantly increased the total DCR. For example, for $V_{\mathrm{EX}}=5 \mathrm{~V}$ the DCR due to BTBT in the n-enhancement region was only $3 \times 10^{-2} \mathrm{~Hz}$ while the perimeter increased the DCR to $900 \mathrm{~Hz}$. The simulations predict that a modification of the n-doped region of the PureB SPADs could decrease the DCR down to the $20 \mathrm{~Hz}$ range. This is achieved by increasing the n-epi-layer thickness from $1 \mu \mathrm{m}$ to $3 \mu \mathrm{m}$, and lowering the doping from $10^{15} \mathrm{~cm}^{-3}$ to $10^{14} \mathrm{~cm}^{-3}$.

A high electric field at the vertical pn junction in the active region can also be minimized by modifying the implantation parameters of the $n$-enhancement region. Lowering the donor concentration at the $\mathrm{p}^{+} \mathrm{n}$ junction of the $\mathrm{n}$-enhancement region increases breakdown voltage and lowers the BTBT contribution to the DCR. These are precautions that also would lower the capacitance of the SPAD but increase the series resistance. Therefore, there is a trade-off between speed and DCR that needs to be considered. This can be done via simulations such as those performed in this paper using our software extension developed to simulate individual avalanche events in Sentaurus Device.

\section{ACKNOWLEDGEMENT}

This work has been supported by Croatian Science Foundation (HRZZ), under the project IP-2018-01-5296.

\section{REFERENCES}

[1] Qi, L. and Nanver, L. K., "Conductance Along the Interface Formed by $400{ }^{\circ} \mathrm{C}$ Pure Boron Deposition on Silicon,” IEEE Electron Device Letters 36(2), 102-104 (2015). 
[2] Nanver, L. K., Qi, L., Mohammadi, V., Mok, K. R. M., De Boer, W. B., Golshani, N., Sammak, A., Scholtes, T. L. M., Gottwald, A., Kroth, U. and Scholze, F., "Robust UV/VUV/EUV PureB photodiode detector technology with high CMOS Compatibility," IEEE Journal on Selected Topics in Quantum Electronics 20(6) (2014).

[3] Mohammadi, V., Qi, L., Golshani, N., Mok, C. K. R., de Boer, W. B., Sammak, A., Derakhshandeh, J., van der Cingel, J. and Nanver, L. K., "VUV/low-energy electron Si photodiodes with postmetal $400{ }^{\circ} \mathrm{C}$ PureB deposition," IEEE Electron Device Letters 34(12), 1545-1547 (2013).

[4] Šakić, A., Nanver, L. K., Veen, G. van, Kooijman, K., Vogelsang, P., Scholtes, T. L. M., Boer, W. de, Wien, W. H. A., Milosavljević, S., Heerkens, C. T. H., Knežević, T. and Spee, I., "Versatile silicon photodiode detector technology for scanning electron microscopy with high-efficiency sub-5 keV electron detection," 2010 International Electron Devices Meeting, 31.4.1-31.4.4 (2010).

[5] Qi, L., Mok, K. R. C., Aminian, M., Charbon, E. and Nanver, L. K., "UV-Sensitive Low Dark-Count PureB Single-Photon Avalanche Diode," IEEE Transactions on Electron Devices 61(11), 3768-3774 (2014).

[6] Qi, L., Sluyterman, S., Kooijman, K., Mok, K. R. C. and Nanver, L. K., "PureB single-photon avalanche diodes for low-energy electron detection down to $200 \mathrm{eV}$," Optics Letters 40(3), 300 (2015).

[7] Cova, S., Ghioni, M., Lacaita, A., Samori, C. and Zappa, F., "Avalanche photodiodes and quenching circuits for single-photon detection," Applied optics 35(12), 1956-1976 (1996).

[8] Lewellen, T. K., "Recent developments in PET detector technology," Physics in Medicine and Biology 53(17), R287-R317 (2008).

[9] Beer, M., Thattil, C., F. Haase, J., Ruskowski, J., Brockherde, W. and Kokozinski, R., "SPAD-Based LiDAR Sensor in $0.35 \mu \mathrm{m}$ Automotive CMOS with Variable Background Light Rejection," Proceedings 2(13), 749 (2018).

[10] Hopman, P. I., Boettcher, P. W., Candell, L. M., Glettler, J. B., Shoup, R. and Zogbi, G., "An end-to-end demonstration of a receiver array based free-space photon counting communications link," 31 August 2006, $63040 \mathrm{H}$.

[11] Shaw, G. A., Siegel, A. M., Model, J. and Greisokh, D., "Recent progress in short-range ultraviolet communication," presented at Defense and Security, 27 May 2005, Orlando, FL, 214-225.

[12] Xu, Z., Chen, G., Abou-Galala, F. and Leonardi, M., "Experimental performance evaluation of non-line-of-sight ultraviolet communication systems," presented at Optical Engineering + Applications, 13 September 2007, San Diego, CA, 67090Y.

[13] McIntyre, R. J., "On the avalanche initiation probability of avalanche diodes above the breakdown voltage," IEEE Transactions on Electron Devices 20(7), 637-641 (1973).

[14] Synopsys., [Sentaurus Device User Guide], Synopsys, Mountain View, CA, USA (2016).

[15] Knežević, T., Nanver, L. K. and Suligoj, T., "2D dark-count-rate modeling of PureB single-photon avalanche diodes in a TCAD environment," Physics and Simulation of Optoelectronic Devices XXVI 10526, 105261K, International Society for Optics and Photonics (2018).

[16] Knežević, T. and Suligoj, T., "Examination of the InP/InGaAs single-photon avalanche diodes by establishing a new TCAD-based simulation environment," Simulation of Semiconductor Processes and Devices (SISPAD), 2016 International Conference on, 57-60, IEEE (2016).

[17] Oldham, W. G., Samuelson, R. R. and Antognetti, P., "Triggering phenomena in avalanche diodes," IEEE Transactions on electron devices 19(9), 1056-1060 (1972).

[18] Qi, L., Mok, K. R. C., Aminian, M., Charbon, E. and Nanver, L. K., "Fabrication of low dark-count PureB singlephoton avalanche diodes," Microelectronics Technology and Devices (SBMicro), 2014 29th Symposium on, 14, IEEE (2014).

[19] Synopsys., [Sentaurus Process User Guide], Synopsys, Mountain View, CA, USA (2016).

[20] Vecchi, M. C. and Rudan, M., "Modeling electron and hole transport with full-band structure effects by means of the spherical-harmonics expansion of the BTE," IEEE Transactions on Electron Devices 45(1), 230-238 (1998).

[21] Hurkx, G. A. M., "On the modelling of tunnelling currents in reverse-biased p-n junctions," Solid-State Electronics 32(8), 665-668 (1989).

[22] Logan, R. A. and Chynoweth, A. G., "Effect of degenerate semiconductor band structure on current-voltage characteristics of silicon tunnel diodes," Physical Review 131(1), 89 (1963). 